Research Article

\title{
Strong Convergence of Modified Algorithms Based on the Regularization for the Constrained Convex Minimization Problem
}

\author{
Ming Tian ${ }^{1,2}$ and Jun-Ying Gong ${ }^{1}$ \\ ${ }^{1}$ College of Science, Civil Aviation University of China, Tianjin 300300, China \\ ${ }^{2}$ Tianjin Key Laboratory for Advanced Signal Processing, Civil Aviation University of China, Tianjin 300300, China
}

Correspondence should be addressed to Ming Tian; tianming1963@126.com

Received 13 May 2014; Revised 31 August 2014; Accepted 1 September 2014; Published 28 October 2014

Academic Editor: Uno Hämarik

Copyright (C) 2014 M. Tian and J.-Y. Gong. This is an open access article distributed under the Creative Commons Attribution License, which permits unrestricted use, distribution, and reproduction in any medium, provided the original work is properly cited.

As is known, the regularization method plays an important role in solving constrained convex minimization problems. Based on the idea of regularization, implicit and explicit iterative algorithms are proposed in this paper and the sequences generated by the algorithms can converge strongly to a solution of the constrained convex minimization problem, which also solves a certain variational inequality. As an application, we also apply the algorithm to solve the split feasibility problem.

\section{Introduction}

Assume that $H$ is a Hilbert space with inner product $\langle\cdot\rangle$ and norm $\|\cdot\|$ induced by its inner product. Let $C$ be a nonempty, closed, and convex subset of $H$. Recall that the projection from $H$ onto $C$, denoted by $\operatorname{Proj}_{C}$, assigns, to each $x \in H$, the unique point $\operatorname{Proj}_{C} x \in C$ with the property

$$
\left\|x-\operatorname{Proj}_{C} x\right\|=\inf \{\|x-y\|: y \in C\} .
$$

Below we introduce some nonlinear operators. Let $T, A$ : $H \rightarrow H$ be nonlinear operators.

(i) $T$ is $L$-Lipschitzian if $\|T x-T y\| \leq L\|x-y\|$, for all $x, y \in H$, where $L \geq 0$ is a constant.

In particular, if $L \in[0,1)$, then $T$ is called a contraction on $H$; if $L=1$, then $T$ is called a nonexpansive mapping on $H$. We know that the projection is nonexpansive.

(ii) $A$ is monotone if, for all $x, y \in H$,

$$
\langle x-y, A x-A y\rangle \geq 0 \text {. }
$$

(iii) Given number $\beta>0, A$ is said to be $\beta$-strongly monotone, if

$$
\langle x-y, A x-A y\rangle \geq \beta\|x-y\|^{2}, \quad x, y \in H .
$$

(iv) Given number $v>0, A$ is said to be $v$-inverse strongly monotone $(v$-ism) if

$$
\langle x-y, A x-A y\rangle \geq v\|A x-A y\|^{2}, \quad x, y \in H .
$$

If $T$ is nonexpansive, then $I-T$ is monotone.

We know that the gradient-projection algorithm can be used to solve the constrained convex minimization problem. Let us recall the concrete analysis below. Consider the following constrained convex minimization problem:

$$
\min _{x \in C} f(x),
$$

where $f: C \rightarrow \mathbb{R}$ is a real-valued function. Assume that $f$ is Fréchet differentiable; define a sequence $\left\{x_{n}\right\}$ by

$$
x_{n+1}=\operatorname{Proj}_{C}(I-\gamma \nabla f)\left(x_{n}\right), \quad n \geq 0,
$$

where the initial guess is taken from $C$ and the parameter $\gamma$ is a real number which satisfies certain conditions. The convergence of algorithm (6) depends on the property of $\nabla f$. In fact, if $\nabla f$ is only inverse strongly monotone, then algorithm (6) can converge weakly to a solution of the minimization problem (5). In 2011, Xu [1] provided an alternative averaged mapping approach to the gradient-projection 
algorithm; he also constructed a counterexample to prove that algorithm (6) has weak convergence only, in infinitedimensional space. He also provided two modifications to ensure that the gradient-projection algorithms can converge strongly to a solution of (5). More investigations about the gradient-projection algorithm and its important role in solving the constrained convex minimization problem can be seen in [2-11]. Recently, the method has also been applied to solve the split feasibility problems which find application in image reconstruction and the intensity modulated radiation therapy (see [12-17]). However, sometimes the minimization problem has more than one solution, so regularization is needed.

Consider the regularized minimization problem

$$
\min _{x \in C} f_{\alpha}(x):=f(x)+\frac{\alpha}{2}\|x\|^{2}
$$

here $\alpha>0$ is the regularization parameter, and again $f$ is Fréchet differentiable and the gradient $\nabla f$ is $(1 / L)$-ism.

On the gradient-projection method based on the regularization, we have weak convergence result as follows: define a sequence by

$$
x_{n+1}:=\operatorname{Proj}_{C}\left(I-\gamma \nabla f_{\alpha_{n}}\right)\left(x_{n}\right), \quad n \geq 0,
$$

where $\alpha_{n}>0,0<\lambda<2 / L$, and $\sum_{n=1}^{\infty} \alpha_{n}<\infty$. Then the sequence generated by (8) converges weakly to a minimizer of (5) in the setting of infinite-dimensional space (see [17]).

On the other hand, Tian [18] proposed the following iterative method:

$$
x_{n+1}=s_{n} \lambda h\left(x_{n}\right)+\left(I-s_{n} \mu F\right) T x_{n}
$$

where $T$ is a nonexpansive mapping on $H$ with a fixed point, $h$ is a contraction on $H$ with coefficient $0<\alpha<1$, and $F: H \rightarrow$ $H$ is a $k$-Lipschitzian and $\eta$-strongly monotone operator with $k, \eta>0$. Letting $0<\mu<2 \eta / k^{2}, 0<\lambda<\mu\left(\eta-\mu k^{2} / 2\right) / \rho=$ $\tau / \rho$, he proved that the sequence $\left\{x_{n}\right\}$ generated by (9) can converge strongly to a fixed point $\widetilde{x} \in \operatorname{Fix}(T)$, which solves the variational inequality $\langle(\lambda h-\mu F) \tilde{x}, x-\tilde{x}\rangle \leq 0, x \in \operatorname{Fix}(T)$.

Combing the idea of regularization with Tian's iterative scheme, in this paper, we will construct a new algorithm. The algorithm can not only find the minimum-solution of the constrained convex minimization problem by a single step but also ensure the strong convergence. In fact, the sequence generated by the constructed algorithm can converge strongly to a minimizer of the constrained convex minimization problem. The obtained point is also a solution of a certain variational inequality. Then we also apply the constructed algorithm to solve a split feasibility problem.

\section{Preliminaries}

Lemma 1 (see [18]). Let C be a nonempty, closed, convex subset of a real Hilbert space $H$. Let $h: C \rightarrow C$ be a contraction with coefficient $0<\rho<1$ and let $F: C \rightarrow C$ be $k$-Lipschitzian and $\eta$-strongly monotone with $k, \eta>0$. Then, for $0<\lambda<\mu \eta / \rho$,

$$
\begin{array}{r}
\langle x-y,(\mu F-\lambda h) x-(\mu F-\lambda h) y\rangle \geq(\mu \eta-\lambda \rho)\|x-y\|^{2}, \\
\forall x, y \in C .
\end{array}
$$

Lemma 2 (see [19]). Let $C$ be a closed and convex subset of a Hilbert space $H$ and let $T: C \rightarrow C$ be a nonexpansive mapping with $\operatorname{Fix}(T) \neq \emptyset$. If $\left\{x_{n}\right\}_{n=1}^{\infty}$ is a sequence in $C$ weakly converging to $x$ and if $\{(I-T)\}_{n=1}^{\infty}$ converges strongly to $y$, then $(I-T) x=y$.

Lemma 3 (see [20]). Let $C$ be a closed and convex subset of a Hilbert space $H$. Given $x \in H$ and $y \in C$, then $y=\operatorname{Proj}_{C} x$ if and only if there holds the inequality

$$
\langle x-y, y-z\rangle \geq 0, \quad \forall z \in C .
$$

Lemma 4 (see [1]). Assume that $\left\{a_{n}\right\}_{n=0}^{\infty}$ is a sequence of nonnegative real numbers such that

$$
a_{n+1} \leq\left(1-\gamma_{n}\right) a_{n}+\gamma_{n} \delta_{n}+\beta_{n}, \quad n \geq 0,
$$

where $\left\{\gamma_{n}\right\}_{n=0}^{\infty}$ and $\left\{\beta_{n}\right\}_{n=0}^{\infty}$ are sequences in $(0,1)$ and $\left\{\delta_{n}\right\}_{n=0}^{\infty}$ is a sequence in $\mathbb{R}$ such that

(i) $\sum_{n=0}^{\infty} \gamma_{n}=\infty$;

(ii) either $\lim \sup _{n \rightarrow \infty} \delta_{n} \leq 0$ or $\sum_{n=0}^{\infty} \gamma_{n}\left|\delta_{n}\right|<\infty$;

(iii) $\sum_{n=0}^{\infty} \beta_{n}<\infty$.

Then $\lim _{n \rightarrow \infty} a_{n}=0$.

We will use the following notation:

(i) $\rightarrow$ for weak convergence and $\rightarrow$ for strong convergence.

\section{Main Results}

Assume that $H$ is a Hilbert space with inner product $\langle\cdot\rangle$ and norm $\|\cdot\|$ induced by its inner product. Let $C$ be a nonempty, closed, and convex subset of $H$. Assume that the minimization problem (5) is consistent and its solution set is denoted by $S$.

Rewrite the regularized constrained convex minimization problem:

$$
\min _{x \in C} f_{\alpha}(x):=f(x)+\frac{\alpha}{2}\|x\|^{2} .
$$

Recall that $h$ is a $\rho$-contraction on $C$ with $0<\rho<1$, and $F$ is $k$-Lipschitzian and $\eta$-strongly monotone on $C$ with $k, \eta>0$. Given $t \in(0,1)$, assume that $\alpha_{t}$ is continuous with respect to $t$ and that $\alpha_{t}=o(t)(t \rightarrow 0)$; then there exists a constant $D>0$ such that $\left|\alpha_{t} / t\right|<D$ for $t \leq t_{0}$. Let the gradient $\nabla f$ be $(1 / L)$-ism. For each $t \in(0, \min (1,1 / \tau))$, we consider the mapping $X_{t}$ on $C$ defined by

$$
\begin{aligned}
& Y_{t}(x):=t \lambda h(x)+(I-t \mu F) \operatorname{Proj}_{C}\left(I-\gamma \nabla f_{\alpha_{t}}\right)(x), \\
& X_{t}(x):=\operatorname{Proj}_{C} Y_{t}(x)
\end{aligned}
$$

where $0<\mu<2 \eta / k^{2}, 0<\lambda<\mu\left(\eta-\mu k^{2} / 2\right) / \rho=\tau / \rho$, and $0<\gamma<2 / L$. 
We will use the following notation in Lemma 5, Proposition 6, and Theorem 7:

$$
\begin{aligned}
T_{\alpha_{t}} & :=\operatorname{Proj}_{C}\left(I-\gamma \nabla f_{\alpha_{t}}\right) ; \\
T & :=\operatorname{Proj}_{C}(I-\gamma \nabla f) .
\end{aligned}
$$

Lemma 5. There exists an implicit algorithm $\left\{x_{t}\right\}$; here $x_{t}$ is the fixed point of $X_{t}$; that is,

$$
x_{t}=X_{t}\left(x_{t}\right)=\operatorname{Proj}_{C} Y_{t}\left(x_{t}\right),
$$

where $X_{t}, Y_{t}$ are defined by (14).

Proof. Below we will show that $X_{t}$ is a contraction. Indeed, we have

$$
\begin{aligned}
\|(I- & t \mu F) T_{\alpha_{t}}(x)-(I-t \mu F) T_{\alpha_{t}}(y) \|^{2} \\
= & \left\|T_{\alpha_{t}}(x)-T_{\alpha_{t}}(y)\right\|^{2}+t^{2} \mu^{2}\left\|F T_{\alpha_{t}}(x)-F T_{\alpha_{t}}(y)\right\|^{2} \\
& \quad-2 t \mu\left\langle T_{\alpha_{t}}(x)-T_{\alpha_{t}}(y), F T_{\alpha_{t}}(x)-F T_{\alpha_{t}}(y)\right\rangle \\
\leq & \left\|T_{\alpha_{t}}(x)-T_{\alpha_{t}}(y)\right\|^{2}+t^{2} \mu^{2} k^{2}\left\|T_{\alpha_{t}}(x)-T_{\alpha_{t}}(y)\right\|^{2} \\
& -2 t \mu \eta\left\|T_{\alpha_{t}}(x)-T_{\alpha_{t}}(y)\right\|^{2} \\
\leq & \left(1-\frac{t \mu\left(2 \eta-t \mu k^{2}\right)}{2}\right)^{2}\left\|T_{\alpha_{t}}(x)-T_{\alpha_{t}}(y)\right\|^{2} \\
\leq & (1-t \tau)^{2}\|x-y\|^{2} .
\end{aligned}
$$

It follows easily that

$$
\begin{aligned}
& \left\|X_{t}(x)-X_{t}(y)\right\| \\
= & \left\|\operatorname{Proj}_{C} Y_{t}(x)-\operatorname{Proj}_{C} Y_{t}(y)\right\| \\
\leq & \left\|Y_{t}(x)-Y_{t}(y)\right\| \\
= & \left\|t \lambda h(x)+(I-t \mu F) T_{\alpha_{t}}(x)-t \lambda h(y)-(I-t \mu F) T_{\alpha_{t}}(y)\right\| \\
\leq & \|t \lambda h(x)-t \lambda h(y)\| \\
& +\left\|(I-t \mu F) T_{\alpha_{t}}(x)-(I-t \mu F) T_{\alpha_{t}}(y)\right\| \\
\leq & (1-t(\tau-\lambda \rho))\|x-y\| .
\end{aligned}
$$

Hence $X_{t}$ has a unique fixed point; we denote it by $x_{t}$, which uniquely solves the fixed point equation

$$
x_{t}=\operatorname{Proj}_{C} Y_{t}\left(x_{t}\right) \text {; }
$$

here $Y_{t}\left(x_{t}\right)=t \lambda h\left(x_{t}\right)+(I-t \mu F) \operatorname{Proj}_{C}\left(I-\gamma \nabla f_{\alpha_{t}}\right)\left(x_{t}\right)$.

Proposition 6. Let $x_{t}$ be defined by (19):

(i) $\left\{x_{t}\right\}$ is bounded for $t \in(0,1)$.

(ii) $\lim _{t \rightarrow 0}\left\|x_{t}-\operatorname{Proj}_{C}\left(I-\gamma \nabla f_{\alpha_{t}}\right)\left(x_{t}\right)\right\|=0$.

(iii) $x_{t}$ defines a continuous curve from $(0,1 / \tau)$ into $C$.
Proof. (i) For $\bar{x} \in S$, we have

$$
\begin{aligned}
& \left\|x_{t}-\bar{x}\right\| \\
& =\left\|\operatorname{Proj}_{C} Y_{t}\left(x_{t}\right)-\operatorname{Proj}_{C}(\bar{x})\right\| \\
& \leq\left\|Y_{t}\left(x_{t}\right)-\bar{x}\right\| \\
& =\left\|t \lambda h\left(x_{t}\right)+(I-t \mu F) T_{\alpha_{t}}\left(x_{t}\right)-\bar{x}\right\| \\
& =\| t \lambda h\left(x_{t}\right)-t \lambda h(\bar{x})+(I-t \mu F) T_{\alpha_{t}}\left(x_{t}\right) \\
& -(I-t \mu F) T_{\alpha_{t}}(\bar{x})+(I-t \mu F) T_{\alpha_{t}}(\bar{x}) \\
& -(I-t \mu F) T(\bar{x})+t \lambda h(\bar{x})-t \mu F(\bar{x}) \| \\
& \leq\left\|t \lambda h\left(x_{t}\right)-t \lambda h(\bar{x})\right\| \\
& +\left\|(I-t \mu F) T_{\alpha_{t}}\left(x_{t}\right)-(I-t \mu F) T_{\alpha_{t}}(\bar{x})\right\| \\
& +\left\|(I-t \mu F) T_{\alpha_{t}}(\bar{x})-(I-t \mu F) T(\bar{x})\right\| \\
& +t\|\lambda h(\bar{x})-\mu F(\bar{x})\| \\
& \leq t \lambda \rho\left\|x_{t}-\bar{x}\right\|+(1-t \tau)\left\|x_{t}-\bar{x}\right\| \\
& +(1+t \mu k) \gamma \alpha_{t}\|\bar{x}\|+t\|\lambda h(\bar{x})-\mu F(\bar{x})\| \\
& \leq(1-t(\tau-\lambda \rho))\left\|x_{t}-\bar{x}\right\|+(1+\mu k) \gamma \alpha_{t}\|\bar{x}\| \\
& +t\|\lambda h(\bar{x})-\mu F(\bar{x})\| \\
& \leq(1-t(\tau-\lambda \rho))\left\|x_{t}-\bar{x}\right\|+(1+\mu k) \gamma t D\|\bar{x}\| \\
& +t\|\lambda h(\bar{x})-\mu F(\bar{x})\| \text {. }
\end{aligned}
$$

It follows that $\left\|x_{t}-\bar{x}\right\| \leq(\|\lambda h(\bar{x})-\mu F(\bar{x})\|+(1+\mu k) \gamma D\|\bar{x}\|) /$ $(\tau-\lambda \rho)$. Hence, $\left\{x_{t}\right\}$ is bounded.

(ii) We can easily see that

$$
\begin{aligned}
\| x_{t}- & \operatorname{Proj}_{C}\left(I-\gamma \nabla f_{\alpha_{t}}\left(x_{t}\right)\right) \| \\
& =\left\|\operatorname{Proj}_{C} Y_{t}\left(x_{t}\right)-\operatorname{Proj}_{C} T_{\alpha_{t}}\left(x_{t}\right)\right\| \\
& \leq\left\|Y_{t}\left(x_{t}\right)-T_{\alpha_{t}}\left(x_{t}\right)\right\| \\
& =\left\|t \lambda h\left(x_{t}\right)+(I-t \mu F) T_{\alpha_{t}}\left(x_{t}\right)-T_{\alpha_{t}}\left(x_{t}\right)\right\| \\
& =t\left\|\lambda h\left(x_{t}\right)-\mu F \operatorname{Proj}_{C}\left(I-\gamma \nabla f_{\alpha_{t}}\right)\left(x_{t}\right)\right\| \\
& \leq t\left(\lambda\left\|h\left(x_{t}\right)\right\|+\mu\left\|F \operatorname{Proj}_{C}\left(I-\gamma \nabla f_{\alpha_{t}}\right)\left(x_{t}\right)\right\|\right) .
\end{aligned}
$$

The boundedness of $\left\{x_{t}\right\}$ implies that $\left\{h\left(x_{t}\right)\right\}$ and $\left\{F \operatorname{Proj}_{C}(I-\right.$ $\left.\left.\gamma \nabla f_{\alpha}\right)\left(x_{t}\right)\right\}$ are also bounded. Hence (ii) follows.

(iii) For $t, t_{0} \in(0,1 / \tau)$, we have

$$
\begin{aligned}
& \left\|x_{t}-x_{t_{0}}\right\| \\
& \quad=\left\|\operatorname{Proj}_{C} Y_{t}\left(x_{t}\right)-\operatorname{Proj}_{C} Y_{t_{0}}\left(x_{t_{0}}\right)\right\| \\
& \quad \leq\left\|Y_{t}\left(x_{t}\right)-Y_{t_{0}}\left(x_{t_{0}}\right)\right\| \\
& \quad=\| t \lambda h\left(x_{t}\right)+(I-t \mu F) T_{\alpha_{t}}\left(x_{t}\right)-t_{0} \lambda h\left(x_{t_{0}}\right)
\end{aligned}
$$




$$
\begin{aligned}
& -\left(I-t_{0} \mu F\right) T_{\alpha_{t_{0}}}\left(x_{t_{0}}\right) \| \\
\leq & \left|t-t_{0}\right| \lambda\left\|h\left(x_{t}\right)\right\|+t_{0} \lambda\left\|h\left(x_{t}\right)-h\left(x_{t_{0}}\right)\right\| \\
& +\left\|(I-t \mu F) T_{\alpha_{t}}\left(x_{t}\right)-\left(I-t_{0} \mu F\right) T_{\alpha_{t_{0}}}\left(x_{t_{0}}\right)\right\| \\
\leq & \left|t-t_{0}\right| \lambda\left\|h\left(x_{t}\right)\right\|+t_{0} \lambda \rho\left\|x_{t}-x_{t_{0}}\right\| \\
& +\left\|(I-t \mu F) T_{\alpha_{t}}\left(x_{t}\right)-\left(I-t_{0} \mu F\right) T_{\alpha_{t_{0}}}\left(x_{t_{0}}\right)\right\| \\
& +\left\|\left(I-t_{0} \mu F\right) T_{\alpha_{t_{0}}}\left(x_{t}\right)-\left(I-t_{0} \mu F\right) T_{\alpha_{t_{0}}}\left(x_{t}\right)\right\| \\
\leq & \left|t-t_{0}\right| \lambda\left\|h\left(x_{t}\right)\right\|+t_{0} \lambda \rho\left\|x_{t}-x_{t_{0}}\right\| \\
& +\left(1-t_{0} \tau\right)\left\|x_{t}-x_{t_{0}}\right\| \\
& +\left\|(I-t \mu F) T_{\alpha_{t}}\left(x_{t}\right)-\left(I-t_{0} \mu F\right) T_{\alpha_{t_{0}}}\left(x_{t}\right)\right\| \\
& +\left|\alpha_{t}-\alpha_{t_{0}}\right| \gamma\left\|x_{t}\right\|+\left|t-t_{0}\right| \mu\left\|F T_{\alpha_{t}}\left(x_{t}\right)\right\| \\
\leq & \left|t-t_{0}\right| \lambda\left\|h\left(x_{t}\right)\right\|+t_{0} \lambda \rho\left\|x_{t}-x_{t_{0}}\right\| \\
& +\left(1-t_{0} \tau\right)\left\|x_{t}-x_{t_{0}}\right\|+\left\|T_{\alpha_{t}}\left(x_{t}\right)-T_{\alpha_{t_{0}}}\left(x_{t}\right)\right\| \\
\leq & t \mu F T_{\alpha_{t}}\left(x_{t}\right)-t_{0} \mu F T_{\alpha_{t_{0}}}\left(x_{t}\right) \| \\
& +\lambda h\left(x_{t}\right)\left\|+\left(1-t_{0}(\tau-\lambda \rho)\right)\right\| x_{t}-x_{t_{0}} \|
\end{aligned}
$$

Hence, $\left\|x_{t}-x_{t_{0}}\right\| \leq\left(\left(\lambda\left\|h\left(x_{t}\right)\right\|+\mu\left\|F \operatorname{Proj}_{C}\left(I-\gamma \nabla f_{\alpha_{t}}\right)\left(x_{t}\right)\right\|\right) /\right.$ $\left.t_{0}(\tau-\lambda \rho)\right)\left|t-t_{0}\right|+\left(\left(1+t_{0} \mu k\right) \gamma\left\|x_{t}\right\| / t_{0}(\tau-\lambda \rho)\right)\left|\alpha_{t}-\alpha_{t_{0}}\right|$. Noting that $\alpha_{t}$ is continuous with respect to $t$, we get $x_{t} \rightarrow x_{t_{0}}$ as $t \rightarrow t_{0}$, and therefore $x_{t}$ is continuous.

Theorem 7. Let $x_{t}$ be defined by (19). Then $x_{t}$ converges in norm, as $t \rightarrow 0$, to a minimizer $x^{*}$ of (5), which solves the following variational inequality:

$$
\left\langle(\mu F-\lambda h) x^{*}, \tilde{x}-x^{*}\right\rangle \geq 0, \quad \forall \tilde{x} \in S .
$$

Proof. It follows from Lemma 1 that the variational inequality (23) has only one solution $x^{*} \in S$. To prove convergence we will use Lemma 3 in the following calculations. It holds that

$$
\begin{aligned}
& \left\|x_{t}-\tilde{x}\right\|^{2} \\
& =\left\langle x_{t}-\tilde{x}, x_{t}-\tilde{x}\right\rangle \\
& =\left\langle\operatorname{Proj}_{C} y_{t}-\tilde{x}, x_{t}-\tilde{x}\right\rangle \\
& =\left\langle\operatorname{Proj}_{C} y_{t}-y_{t}+y_{t}-\tilde{x}, x_{t}-\tilde{x}\right\rangle \\
& =\left\langle\operatorname{Proj}_{C} y_{t}-y_{t}, \operatorname{Proj}_{C} y_{t}-\tilde{x}\right\rangle+\left\langle y_{t}-\tilde{x}, x_{t}-\tilde{x}\right\rangle \\
& \leq\left\langle y_{t}-\tilde{x}, x_{t}-\tilde{x}\right\rangle \\
& =\left\langle t \lambda h\left(x_{t}\right)+(I-t \mu F) T_{\alpha_{t}}\left(x_{t}\right)-\tilde{x}, x_{t}-\tilde{x}\right\rangle
\end{aligned}
$$

$$
\begin{aligned}
= & \left\langle t \lambda h\left(x_{t}\right)-t \lambda h(\tilde{x}), x_{t}-\tilde{x}\right\rangle \\
& +\left\langle(I-t \mu F) T_{\alpha_{t}}\left(x_{t}\right)-(I-t \mu F) T_{\alpha_{t}}(\tilde{x}), x_{t}-\tilde{x}\right\rangle \\
& +\left\langle(I-t \mu F) T_{\alpha_{t}}(\tilde{x})-(I-t \mu F) T(\tilde{x}), x_{t}-\tilde{x}\right\rangle \\
& +\left\langle t \lambda h(\tilde{x})-t \mu F(\tilde{x}), x_{t}-\tilde{x}\right\rangle \\
\leq & t \lambda \rho\left\|x_{t}-\tilde{x}\right\|^{2}+(1-t \tau)\left\|x_{t}-\tilde{x}\right\|^{2} \\
& +(1+t \mu k) \gamma \alpha_{t}\|\tilde{x}\|\left\|x_{t}-\tilde{x}\right\|+t\left\langle\lambda h(\tilde{x})-\mu F(\tilde{x}), x_{t}-\tilde{x}\right\rangle \\
\leq & (1-t(\tau-\lambda \rho))\left\|x_{t}-\tilde{x}\right\|^{2} \\
& +(1+\mu k) \gamma \alpha_{t}\|\tilde{x}\|\left\|x_{t}-\tilde{x}\right\| \\
& +t\left\langle\lambda h(\tilde{x})-\mu F(\tilde{x}), x_{t}-\tilde{x}\right\rangle .
\end{aligned}
$$

Hence,

$$
\begin{aligned}
\left\|x_{t}-\tilde{x}\right\|^{2} \leq & \frac{1}{\tau-\lambda \rho}\left\langle\lambda h(\tilde{x})-\mu F(\tilde{x}), x_{t}-\tilde{x}\right\rangle \\
& +\frac{\alpha_{t}}{t} \frac{(1+\mu k) \gamma}{\tau-\lambda \rho}\left\|x_{t}-\tilde{x}\right\| .
\end{aligned}
$$

Since $x_{t}$ is bounded for $t \in(0,1)$, and $\alpha_{t}=o(t)(t \rightarrow \infty)$, we see that if $t_{n}$ is a sequence in $(0,1)$ such that $t_{n} \rightarrow 0$ and $x_{t_{n}} \rightarrow \bar{x}$, then, by (25), $x_{t_{n}} \rightarrow \bar{x}$. We may further assume that $\alpha_{t_{n}} \rightarrow 0$. It turns out that

$$
\begin{aligned}
& \left\|x_{t_{n}}-\operatorname{Proj}_{C}(I-\gamma \nabla f) x_{t_{n}}\right\| \\
& \quad \leq\left\|x_{t_{n}}-\operatorname{Proj}_{C}\left(I-\gamma \nabla f_{\alpha_{t_{n}}}\right) x_{t_{n}}\right\|+\gamma \alpha_{t_{n}}\left\|x_{t_{n}}\right\| .
\end{aligned}
$$

From the boundedness of $\left\{x_{t}\right\}$ and $\lim _{t \rightarrow 0} \| x_{t}-\operatorname{Proj}_{C}(I-$ $\left.\gamma \nabla f_{\alpha_{t}}\right) x_{t}-x_{t} \|=0$, we conclude that

$$
\lim _{n \rightarrow \infty}\left\|x_{t_{n}}-\operatorname{Proj}_{C}(I-\gamma \nabla f) x_{t_{n}}\right\|=0
$$

It then follows from Lemma 2 that $\bar{x}=\operatorname{Proj}_{C}(I-\gamma \nabla f) \bar{x}$. This shows that $\bar{x} \in S$.

We next prove that $\bar{x}$ is a solution of the variational inequality (23). Since $\operatorname{Proj}_{C}\left(I-\gamma \nabla f_{\alpha_{t}}\right)$ is nonexpansive, we see that $I-\operatorname{Proj}_{C}\left(I-\gamma \nabla f_{\alpha_{t}}\right)$ is monotone. By (19), we have

$$
x_{t}=\operatorname{Proj}_{C} Y_{t}\left(x_{t}\right)-Y_{t}\left(x_{t}\right)+t \lambda h\left(x_{t}\right)+(I-t \mu F) T \alpha_{t}\left(x_{t}\right) ;
$$


then it follows that

$$
\begin{aligned}
& \lambda h\left(x_{t}\right)=-\frac{1}{t}\left[Y_{t}\left(x_{t}\right)-Y_{t}\left(x_{t}\right)+(I-t \mu F) T \alpha_{t}\left(x_{t}\right)-x_{t}\right], \\
& (\mu F-\lambda h)\left(x_{t}\right) \\
& =\mu F\left(x_{t}\right) \\
& +\frac{1}{t}\left[\operatorname{Proj}_{C} Y_{t}\left(x_{t}\right)-Y_{t}\left(x_{t}\right)+(I-t \mu F) T \alpha_{t}\left(x_{t}\right)-x_{t}\right], \\
& \left\langle(\mu F-\lambda h)\left(x_{t}\right), x_{t}-\tilde{x}\right\rangle \\
& =\left\langle\mu F\left(x_{t}\right)+\frac{1}{t}\left[\operatorname{Proj}_{C} Y_{t}\left(x_{t}\right)-Y_{t}\left(x_{t}\right)\right.\right. \\
& \left.\left.+(I-t \mu F) T \alpha_{t}\left(x_{t}\right)-x_{t}\right], x_{t}-\tilde{x}\right\rangle \\
& =\frac{1}{t}\left\langle\operatorname{Proj}_{C} Y_{t}\left(x_{t}\right)-Y_{t}\left(x_{t}\right), x_{t}-\tilde{x}\right\rangle \\
& +\left\langle\mu F\left(x_{t}\right)+\frac{1}{t}\left[(I-t \mu F) T \alpha_{t}\left(x_{t}\right)-x_{t}\right], x_{t}-\tilde{x}\right\rangle \\
& \leq\left\langle\mu F\left(x_{t}\right)+\frac{1}{t}\left[(I-t \mu F) T \alpha_{t}\left(x_{t}\right)-x_{t}\right], x_{t}-\tilde{x}\right\rangle \\
& =\mu\left\langle F\left(x_{t}\right)-F T_{\alpha_{t}}\left(x_{t}\right), x_{t}-\tilde{x}\right\rangle \\
& -\frac{1}{t}\left\langle\left(I-T_{\alpha_{t}}\right)\left(x_{t}\right), x_{t}-\tilde{x}\right\rangle \\
& =\mu\left\langle F\left(x_{t}\right)-F T_{\alpha_{t}}\left(x_{t}\right), x_{t}-\tilde{x}\right\rangle \\
& -\frac{1}{t}\left\langle\left(I-T_{\alpha_{t}}\right)\left(x_{t}\right)-\left(I-T_{\alpha_{t}}\right)(\tilde{x})\right. \\
& \left.-T_{\alpha_{t}}(\tilde{x})+T(\tilde{x}), x_{t}-\tilde{x}\right\rangle \\
& =\mu\left\langle F\left(x_{t}\right)-F T_{\alpha_{t}}\left(x_{t}\right), x_{t}-\tilde{x}\right\rangle \\
& -\frac{1}{t}\left\langle\left(I-T \alpha_{t}\right)\left(x_{t}\right)-\left(I-T_{\alpha_{t}}\right)(\tilde{x}), x_{t}-\tilde{x}\right\rangle \\
& +\frac{1}{t}\left\langle T_{\alpha_{t}}(\tilde{x})-T(\tilde{x}), x_{t}-\tilde{x}\right\rangle \\
& \leq \mu\left\langle F\left(x_{t}\right)-F T_{\alpha_{t}}\left(x_{t}\right), x_{t}-\tilde{x}\right\rangle \\
& +\frac{1}{t} \alpha_{t} \gamma\|\tilde{x}\|\left\|x_{t}-\tilde{x}\right\| .
\end{aligned}
$$

Taking the limit through $t=t_{n} \rightarrow 0$ ensures that $\bar{x}$ is a solution of the variational inequality. This implies that $\langle(\mu F-$ $\lambda h)(\bar{x}), \tilde{x}-\bar{x}\rangle \geq 0$. Therefore $\bar{x}=x^{*}$.

Finally we consider the explicit version of our algorithm which is

$$
\begin{aligned}
& y_{n}=s_{n} \lambda h\left(x_{n}\right)+\left(I-s_{n} \mu F\right) \operatorname{Proj}_{C}\left(I-\gamma \nabla f_{\alpha_{n}}\right)\left(x_{n}\right), \\
& x_{n+1}=\operatorname{Proj}_{C} y_{n},
\end{aligned}
$$

where the initial guess $x_{0} \in C$ and $\left\{\alpha_{n}\right\}_{n=0}^{\infty}$ and $\left\{s_{n}\right\}_{n=0}^{\infty} \subset(0,1)$ are the parameter sequences satisfying certain conditions.

Theorem 8. Assume that the minimization problem (5) is consistent and let $S$ denote its solution set. Let the gradient $\nabla f$ be $(1 / L)$-ism. Let $F: C \rightarrow C$ be $\eta$-strongly monotone and $k$-Lipschitzian and let $h: C \rightarrow C$ be a contraction with coefficient $0<\rho<1$. Fix a constant $\mu$ satisfying $0<\mu<2 \eta / k^{2}$, a constant $\lambda$ with the property $0<\lambda<\mu\left(\eta-\mu k^{2} / 2\right) / \rho=\tau / \rho$, and a constant $\gamma$ satisfying $0<\gamma<2 /$ L. Let $\left\{x_{n}\right\}_{n=0}^{\infty}$ be generated by the iterative algorithm (30). Assume

$(\mathrm{C} 1) s_{n} \rightarrow 0$;

(C2) $\alpha_{n}=o\left(s_{n}\right)\left(s_{n} \rightarrow 0, n \rightarrow \infty\right)$;

(C3) $\sum_{n=0}^{\infty} s_{n}=\infty$;

(C4) $\sum_{n=0}^{\infty}\left|s_{n+1}-s_{n}\right|<\infty$;

(C5) $\sum_{n=0}^{\infty}\left|\alpha_{n+1}-\alpha_{n}\right|<\infty$.

Then the sequence $\left\{x_{n}\right\}$ converges in norm to $x^{*}$ as defined in Theorem 7.

Proof. We set

$$
\begin{aligned}
T_{\alpha_{n}} & :=\operatorname{Proj}_{C}\left(I-\gamma \nabla f_{\alpha_{n}}\right) \\
T & :=\operatorname{Proj}_{C}(I-\gamma \nabla f) .
\end{aligned}
$$

Since $\alpha_{n}=o\left(s_{n}\right)\left(s_{n} \rightarrow 0, n \rightarrow \infty\right)$, there exists $M>0$ such that $\alpha_{n} / s_{n}<M$ for $n>n_{0}$.

We observe that $\left\{x_{n}\right\}$ is bounded. Indeed, taking a fixed point $p \in S$, we get

$$
\begin{aligned}
\left\|x_{n+1}-p\right\| & \left\|\operatorname{Proj}_{C} y_{n}-\operatorname{Proj}_{C} p\right\| \\
\leq & \left\|y_{n}-p\right\| \\
= & \left\|s_{n} \lambda h\left(x_{n}\right)+\left(I-s_{n} \mu F\right) T_{\alpha_{n}}\left(x_{n}\right)-p\right\| \\
\leq & \left\|s_{n} \lambda h\left(x_{n}\right)-s_{n} \lambda h(p)\right\| \\
& +\left\|\left(I-s_{n} \mu F\right) T_{\alpha_{n}}\left(x_{n}\right)-\left(I-s_{n} \mu F\right) T_{\alpha_{n}}(p)\right\| \\
& +\left\|\left(I-s_{n} \mu F\right) T_{\alpha_{n}}(p)-\left(I-s_{n} \mu F\right) T(p)\right\| \\
& +\left\|s_{n} \lambda h(p)-s_{n} \mu F(p)\right\| \\
\leq & s_{n} \lambda \rho\left\|x_{n}-p\right\|+\left(1-s_{n} \tau\right)\left\|x_{n}-p\right\| \\
& +\left(1+s_{n} \mu k\right) \gamma \alpha_{n}\|p\|+s_{n}\|\lambda h(p)-\mu F(p)\| \\
\leq & \left(1-s_{n}(\tau-\lambda \rho)\right)\left\|x_{n}-p\right\| \\
& +(1+\mu k) \gamma M s_{n}\|p\|+s_{n}\|\lambda h(p)-\mu F(p)\| \\
\leq & \max \left\{\left\|x_{n}-p\right\|, \frac{(1+\mu k) \gamma M\|p\|+\|\lambda h(p)-\mu F(p)\|}{\tau-\lambda \rho}\right\} .
\end{aligned}
$$


By induction $\left\|x_{n}-p\right\| \leq \max \left\{\left\|x_{0}-p\right\|,((1+\mu k) \gamma M\|p\|+\right.$ $\|\lambda h(p)-\mu F(p)\|) /(\tau-\lambda \rho)\}, n \geq 0$, then $\left\{x_{n}\right\}$ is bounded, so are $\left\{h\left(x_{n}\right)\right\}$ and $\left\{F \operatorname{Proj}_{C}\left(I-\gamma \nabla f_{\alpha_{n}}\right)\left(x_{n}\right)\right\}$.

We claim that $\left\|x_{n+1}-x_{n}\right\| \rightarrow 0$.

Because of the boundedness of $\left\{x_{n}\right\}$, it can be easily seen that $\left\{h\left(x_{n}\right)\right\}$ and $\left\{F T_{\alpha_{n}}\left(x_{n}\right)\right\}$ are also bounded. So we can take two constants $N, E$ such that $N \geq \lambda\left\|h\left(x_{n-1}\right)\right\|+$ $\mu\left\|F T_{\alpha_{n}}\left(x_{n-1}\right)\right\|$ and $E \geq(1+\mu k) \gamma\left\|x_{n-1}\right\|$. Consider

$$
\begin{aligned}
& \left\|x_{n+1}-x_{n}\right\| \\
& =\left\|\operatorname{Proj}_{C} y_{n}-\operatorname{Proj}_{C} y_{n-1}\right\| \\
& \leq\left\|y_{n}-y_{n-1}\right\| \\
& =\| s_{n} \lambda h\left(x_{n}\right)+\left(I-s_{n} \mu F\right) T_{\alpha_{n}}\left(x_{n}\right)-s_{n-1} \lambda h\left(x_{n-1}\right) \\
& -\left(I-s_{n-1} \mu F\right) T_{\alpha_{n-1}}\left(x_{n-1}\right) \| \\
& \leq\left\|s_{n} \lambda h\left(x_{n}\right)-s_{n} \lambda h\left(x_{n-1}\right)\right\| \\
& +\left\|s_{n} \lambda h\left(x_{n-1}\right)-s_{n-1} \lambda h\left(x_{n-1}\right)\right\| \\
& +\left\|\left(I-s_{n} \mu F\right) T_{\alpha_{n}}\left(x_{n}\right)-\left(I-s_{n-1} \mu F\right) T_{\alpha_{n-1}}\left(x_{n-1}\right)\right\| \\
& \leq s_{n} \lambda \rho\left\|x_{n}-x_{n-1}\right\|+\left|s_{n}-s_{n-1}\right| \lambda\left\|h\left(x_{n-1}\right)\right\| \\
& +\left\|\left(I-s_{n} \mu F\right) T_{\alpha_{n}}\left(x_{n}\right)-\left(I-s_{n-1} \mu F\right) T_{\alpha_{n-1}}\left(x_{n-1}\right)\right\| \\
& \leq\left(1-s_{n}(\tau-\lambda \rho)\right)\left\|x_{n}-x_{n-1}\right\| \\
& +\left|s_{n}-s_{n-1}\right| \lambda\left\|h\left(x_{n-1}\right)\right\| \\
& +\left\|T_{\alpha_{n}}\left(x_{n-1}\right)-T_{\alpha_{n-1}}\left(x_{n-1}\right)\right\| \\
& +\left\|s_{n} \mu F T_{\alpha_{n}}\left(x_{n-1}\right)-s_{n-1} \mu F T_{\alpha_{n-1}}\left(x_{n-1}\right)\right\| \\
& \leq\left(1-s_{n}(\tau-\lambda \rho)\right)\left\|x_{n}-x_{n-1}\right\| \\
& +\left|s_{n}-s_{n-1}\right| \lambda\left\|h\left(x_{n-1}\right)\right\| \\
& +\gamma\left|\alpha_{n}-\alpha_{n-1}\right|\left\|x_{n-1}\right\| \\
& +\left\|s_{n} \mu F T_{\alpha_{n}}\left(x_{n-1}\right)-s_{n-1} \mu F T_{\alpha_{n-1}}\left(x_{n-1}\right)\right\| \\
& \leq\left(1-s_{n}(\tau-\lambda \rho)\right)\left\|x_{n}-x_{n-1}\right\| \\
& +\left|s_{n}-s_{n-1}\right| \lambda\left\|h\left(x_{n-1}\right)\right\|+\gamma\left|\alpha_{n}-\alpha_{n-1}\right|\left\|x_{n-1}\right\| \\
& +\left|s_{n}-s_{n-1}\right| \mu\left\|F T_{\alpha_{n}}\left(x_{n-1}\right)\right\| \\
& +s_{n-1} \mu k \gamma\left|\alpha_{n}-\alpha_{n-1}\right|\left\|x_{n-1}\right\| \\
& \leq\left(1-s_{n}(\tau-\lambda \rho)\right)\left\|x_{n}-x_{n-1}\right\| \\
& +\left(\lambda\left\|h\left(x_{n-1}\right)\right\|+\mu\left\|F T_{\alpha_{n}}\left(x_{n-1}\right)\right\|\right)\left|s_{n}-s_{n-1}\right| \\
& +(1+\mu k) \gamma\left\|x_{n-1}\right\|\left|\alpha_{n}-\alpha_{n-1}\right| \\
& \leq\left(1-s_{n}(\tau-\lambda \rho)\right)\left\|x_{n}-x_{n-1}\right\|+N\left|s_{n}-s_{n-1}\right| \\
& +E\left|\alpha_{n}-\alpha_{n-1}\right| \text {. }
\end{aligned}
$$

By Lemma 4 , we obtain $\left\|x_{n+1}-x_{n}\right\| \rightarrow 0$.

Next we show that

$$
\left\|x_{n}-\operatorname{Proj}_{C}(I-\gamma \nabla f) x_{n}\right\| \longrightarrow 0,
$$

$$
\begin{aligned}
\| x_{n} & -\operatorname{Proj}_{C}(I-\gamma \nabla f) x_{n} \| \\
\leq & \left\|x_{n}-x_{n+1}\right\|+\left\|x_{n+1}-\operatorname{Proj}_{C}(I-\gamma \nabla f) x_{n}\right\| \\
\leq & \left\|x_{n}-x_{n+1}\right\| \\
& +\left\|\operatorname{Proj}_{C} y_{n}-\operatorname{Proj}_{C}\left[\operatorname{Proj}_{C}(I-\gamma \nabla f) x_{n}\right]\right\| \\
\leq & \left\|x_{n}-x_{n+1}\right\|+\left\|y_{n}-T\left(x_{n}\right)\right\| \\
\leq & \left\|x_{n}-x_{n+1}\right\| \\
& +\left\|s_{n} \lambda h\left(x_{n}\right)+\left(I-s_{n} \mu F\right) T_{\alpha_{n}}\left(x_{n}\right)-T\left(x_{n}\right)\right\| \\
\leq & \left\|x_{n}-x_{n+1}\right\| \\
& +s_{n}\left\|\lambda h\left(x_{n}\right)-\mu F \operatorname{Proj}_{C}\left(I-\gamma \nabla f_{\alpha_{n}}\right)\left(x_{n}\right)\right\| \\
& +\gamma \alpha_{n}\left\|x_{n}\right\| \longrightarrow 0 .
\end{aligned}
$$

We next show that

$$
\lim _{n \rightarrow \infty} \sup \left\langle x_{n}-x^{*},(\gamma h-\mu F) x^{*}\right\rangle \leq 0
$$

where $x^{*}$ is obtained in Theorem 7. Indeed, take a subsequence $\left\{x_{n_{k}}\right\}$ of $\left\{x_{n}\right\}$ such that

$$
\begin{aligned}
\lim _{n \rightarrow \infty} & \sup \left\langle x_{n}-x^{*},(\gamma h-\mu F) x^{*}\right\rangle \\
& =\lim _{k \rightarrow \infty}\left\langle x_{n_{k}}-x^{*},(\gamma h-\mu F) x^{*}\right\rangle .
\end{aligned}
$$

Since the sequence $\left\{x_{n}\right\}$ is bounded, we may assume that $x_{n_{k}} \rightarrow z$; it follows from Lemma 2 and (34) that $z \in S$. Then we obtain $\lim _{k \rightarrow \infty}\left\langle x_{n_{k}}-x^{*},(\gamma h-\mu F) x^{*}\right\rangle=\left\langle z-x^{*},(\gamma h-\right.$ $\left.\mu F) x^{*}\right\rangle \leq 0$. By (37) we know that $\lim _{n \rightarrow \infty} \sup \left\langle x_{n}-x^{*},(\gamma h-\right.$ $\left.\mu F) x^{*}\right\rangle \leq 0$.

We finally show that $x_{n} \rightarrow x^{*}$. We have, using Lemma 3,

$$
\begin{aligned}
\| & x_{n+1}-x^{*} \|^{2} \\
= & \left\langle x_{n+1}-x^{*}, x_{n+1}-x^{*}\right\rangle \\
= & \left\langle\operatorname{Proj}_{C} y_{n}-y_{n}+y_{n}-x^{*}, x_{n+1}-x^{*}\right\rangle \\
= & \left\langle\operatorname{Proj}_{C} y_{n}-y_{n}, x_{n+1}-x^{*}\right\rangle+\left\langle y_{n}-x^{*}, x_{n+1}-x^{*}\right\rangle \\
\leq & \left\langle y_{n}-x^{*}, x_{n+1}-x^{*}\right\rangle \\
= & \left\langle s_{n} \lambda h\left(x_{n}\right)+\left(I-s_{n} \mu F\right) T_{\alpha_{n}}\left(x_{n}\right)-x^{*}, x_{n+1}-x^{*}\right\rangle \\
= & \left\langle s_{n} \lambda h\left(x_{n}\right)-s_{n} \lambda h\left(x^{*}\right), x_{n+1}-x^{*}\right\rangle \\
& +\left\langle s_{n} \lambda h\left(x^{*}\right)-s_{n} \mu F\left(x^{*}\right), x_{n+1}-x^{*}\right\rangle
\end{aligned}
$$




$$
\begin{aligned}
& +\left\langle\left(I-s_{n} \mu F\right) T_{\alpha_{n}}\left(x_{n}\right)-\left(I-s_{n} \mu F\right) T_{\alpha_{n}}\left(x^{*}\right), x_{n+1}-x^{*}\right\rangle \\
& +\left\langle\left(I-s_{n} \mu F\right) T_{\alpha_{n}}\left(x^{*}\right)-\left(I-s_{n} \mu F\right)\left(x^{*}\right), x_{n+1}-x^{*}\right\rangle \\
\leq & s_{n} \lambda \rho\left\|x_{n}-x^{*}\right\|\left\|x_{n+1}-x^{*}\right\| \\
& +s_{n}\left\langle(\lambda h-\mu F) x^{*}, x_{n+1}-x^{*}\right\rangle \\
& +\left(1-s_{n} \tau\right)\left\|x_{n}-x^{*}\right\|\left\|x_{n+1}-x^{*}\right\| \\
& +\left(1+s_{n} \mu k\right) \gamma \alpha_{n}\left\|x^{*}\right\|\left\|x_{n+1}-x^{*}\right\| \\
\leq & \frac{1}{2}\left(1-s_{n}(\tau-\lambda \rho)\right)\left(\left\|x_{n}-x^{*}\right\|^{2}+\left\|x_{n+1}-x^{*}\right\|^{2}\right) \\
& +s_{n}\left\langle(\lambda h-\mu F) x^{*}, x_{n+1}-x^{*}\right\rangle \\
& +(1+\mu k) \gamma \alpha_{n}\left\|x^{*}\right\|\left\|x_{n+1}-x^{*}\right\| .
\end{aligned}
$$

Since $\left\{x_{n}\right\}$ is bounded, we can take a constant $L^{\prime}>0$ such that

$$
L^{\prime} \geq(1+\mu k) \gamma\left\|x^{*}\right\|\left\|x_{n+1}-x^{*}\right\| ;
$$

hence,

$$
\begin{aligned}
\| x_{n+1} & -x^{*} \|^{2} \\
\leq & \frac{1-s_{n}(\tau-\lambda \rho)}{1+s_{n}(\tau-\lambda \rho)}\left\|x_{n}-x^{*}\right\|^{2} \\
& +\frac{2 s_{n}}{1+s_{n}(\tau-\lambda \rho)}\left\langle(\lambda h-\mu F) x^{*}, x_{n+1}-x^{*}\right\rangle \\
& +\frac{2(1+\mu k) \gamma \alpha_{n}\left\|x^{*}\right\|\left\|x_{n+1}-x^{*}\right\|}{1+s_{n}(\tau-\lambda \rho)} \\
\leq & \left(1-s_{n}(\tau-\lambda \rho)\right)\left\|x_{n}-x^{*}\right\|^{2} \\
& +\frac{s_{n}}{1+s_{n}(\tau-\lambda \rho)} \\
& \times\left[2\left\langle(\lambda h-\mu F) x^{*}, x_{n+1}-x^{*}\right\rangle+2 L^{\prime} \frac{\alpha_{n}}{s_{n}}\right]
\end{aligned}
$$

it then follows that

$$
\left\|x_{n+1}-x^{*}\right\| \leq\left(1-s_{n}(\tau-\lambda \rho)\right)\left\|x_{n}-x^{*}\right\|^{2}+s_{n} \delta_{n},
$$

where $\delta_{n}=\left(2\left\langle(\lambda h-\mu F) x^{*}, x_{n+1}-x^{*}\right\rangle+2 L^{\prime}\left(\alpha_{n} / s_{n}\right)\right) /(1+$ $\left.s_{n}(\tau-\lambda \rho)\right)$.

Since $s_{n} \rightarrow 0$ and $\alpha_{n}=o\left(s_{n}\right)\left(s_{n} \rightarrow 0, n \rightarrow \infty\right)$, by (37), we get $\lim _{n \rightarrow \infty}$ sup $\delta_{n} \leq 0$. Applying Lemma 4 to (41) concludes that $x_{n} \rightarrow x^{*}$ as $n \rightarrow \infty$.

\section{An Application}

Since the split feasibility problem (say SFP, for short) was proposed by Censor and Elfving in 1994, it has been widely used in signal processing and image reconstruction, with particular progress in intensity-modulated radiation therapy.
We know that the gradient-projection method plays an important role in solving the SFP. In this section, we provide an application of Theorem 8 to the SFP (see [21, 22]).

The SFP can mathematically be formulated as the problem of finding a point $x$ with the property

$$
x \in C, \quad B x \in Q,
$$

where $C$ and $Q$ are nonempty, closed, and convex subset of Hilbert spaces $H_{1}$ and $H_{2}$, respectively. $B: H_{1} \rightarrow H_{2}$ is a bounded linear operator.

It is clear that $x^{*}$ is a solution to the split feasibility problem if and only if $x^{*} \in C$ and $B x^{*}-\operatorname{Proj}_{C} B x^{*}=0$. We define the proximity function $f$ by

$$
f(x)=\frac{1}{2}\left\|B x-\operatorname{Proj}_{Q} B x\right\|^{2},
$$

and we consider the constrained convex minimization problem

$$
\min _{x \in C} \frac{1}{2}\left\|B x-\operatorname{Proj}_{Q} B x\right\|^{2} .
$$

Then $x^{*}$ solves the split feasibility problem (42) if and only if $x^{*}$ solves the minimization problem (44) with the minimal value equal to 0 . Byrne introduced the so-called $C Q$ algorithm to solve the SFP:

$$
x_{n+1}=\operatorname{Proj}_{C}\left(I-\gamma B^{*}\left(I-\operatorname{Proj}_{Q}\right) B\right) x_{n}, \quad n \geq 0,
$$

where $0<\gamma<2 /\|B\|^{2}$. He obtained that the sequence $x_{n}$ generated by (45) converges weakly to a solution of the SFP.

Now we consider the regularization technique; let

$$
f_{\alpha}(x)=\frac{1}{2}\left\|B x-P_{\mathrm{Q}} B x\right\|^{2}+\frac{\alpha}{2}\|x\|^{2} ;
$$

then we establish the iterative scheme as follows:

$$
\begin{aligned}
y_{n}= & s_{n} \lambda h\left(x_{n}\right) \\
& +\left(I-s_{n} \mu F\right) \operatorname{Proj}_{C}\left(I-\gamma\left(B^{*}\left(I-P_{\mathrm{Q}}\right) B+\alpha_{n} I\right)\right) x_{n}, \\
x_{n+1} & =\operatorname{Proj}_{C} y_{n},
\end{aligned}
$$

where $h: C \rightarrow H$ is a contraction with the coefficient $\rho \in(0,1)$. Let $F: C \rightarrow H$ be $\eta$-strongly monotone and $k$-Lipschitzian.

Theorem 9. Assume that the split feasibility problem (42) is consistent. Let the sequence $\left\{x_{n}\right\}$ be generated by (47). The $\mu$ and $\lambda$ are constants with the same property as in Theorem 8. Fix $0<\gamma<2 /\|B\|^{2}$. The sequences $\left\{\alpha_{n}\right\}_{n=1}^{\infty}$ and $\left\{s_{n}\right\}_{n=1}^{\infty} \subset(0,1)$ are the parameter sequences satisfying conditions (C1)-(C5) in Theorem 8 . Then the sequence $\left\{x_{n}\right\}$ generated by (47) converges strongly to the solution of split feasibility problem (42).

Proof. By the definition of the proximity function $f$, we have

$$
\nabla f=B^{*}\left(I-\operatorname{Proj}_{\mathrm{Q}}\right) B x,
$$

and we show that $\nabla f$ is $1 /\|B\|^{2}$-ism. 
Since $\operatorname{Proj}_{Q}$ is $(1 / 2)$-averaged mapping, then $I-\operatorname{Proj}_{Q}$ is 1-ism:

$$
\begin{aligned}
\langle\nabla f & (x)-\nabla f(y), x-y\rangle-\frac{1}{\|B\|^{2}} \cdot\|\nabla f(x)-\nabla f(y)\|^{2} \\
= & \left\langle B^{*}\left(I-\operatorname{Proj}_{\mathrm{Q}}\right) B x-B^{*}\left(I-\operatorname{Proj}_{\mathrm{Q}}\right) B y, x-y\right\rangle \\
& -\frac{1}{\|B\|^{2}} \cdot\left\|B^{*}\left(I-\operatorname{Proj}_{\mathrm{Q}}\right) B x-B^{*}\left(I-\operatorname{Proj}_{\mathrm{Q}}\right) B y\right\|^{2} \\
= & \left\langle B^{*}\left[\left(I-\operatorname{Proj}_{\mathrm{Q}}\right) B x-\left(I-\operatorname{Proj}_{\mathrm{Q}}\right) B y\right], x-y\right\rangle \\
& -\frac{1}{\|B\|^{2}} \cdot\left\|B^{*}\left[\left(I-\operatorname{Proj}_{\mathrm{Q}}\right) B x-\left(I-\operatorname{Proj}_{\mathrm{Q}}\right) B y\right]\right\|^{2} \\
= & \left\langle\left(I-\operatorname{Proj}_{\mathrm{Q}}\right) B x-\left(I-\operatorname{Proj}_{\mathrm{Q}}\right) B y, B x-B y\right\rangle \\
& -\frac{1}{\|B\|^{2}} \cdot\|B\|^{2}\left\|\left(I-\operatorname{Proj}_{\mathrm{Q}}\right) B x-\left(I-\operatorname{Proj}_{\mathrm{Q}}\right) B y\right\|^{2} \\
\geq & \left\|\left(I-\operatorname{Proj}_{\mathrm{Q}}\right) B x-\left(I-\operatorname{Proj}_{\mathrm{Q}}\right) B y\right\|^{2} \\
& -\left\|\left(I-\operatorname{Proj}_{\mathrm{Q}}\right) B x-\left(I-\operatorname{Proj}_{\mathrm{Q}}\right) B y\right\|^{2} \\
= & 0 .
\end{aligned}
$$

Hence, $\langle\nabla f(x)-\nabla f(y), x-y\rangle \geq 1 /\|B\|^{2} \cdot\|\nabla f(x)-\nabla f(y)\|^{2}$. Let $L=\|B\|^{2}$; then the iterative scheme (47) is equivalent to

$$
\begin{array}{r}
x_{n+1} \\
=\operatorname{Proj}_{C}\left[s_{n} \lambda h\left(x_{n}\right)+\left(I-s_{n} \mu F\right) \operatorname{Proj}_{C}\left(I-\gamma \nabla f_{\alpha_{n}}\right) x_{n}\right], \\
n \geq 0,
\end{array}
$$

where $0<\gamma<2 / L$, and then, due to Theorem 8 , we have the conclusion immediately.

\section{Numerical Result}

In this section, we present the following simple example to judge the numerical performance of our algorithm. We use the algorithm in Theorem 9 to illustrate its realization in solving system of linear equations.

Example 10. In Theorem 9, we assume that $H_{1}=H_{2}=\mathbb{R}^{3}$. Take $h=(1 / 2) I$, where $I$ denotes the $3 \times 3$ identity matrix, and $F=I$ with Lipschitz constant $k=1$ and strongly monotone constant $\eta=1$. Given the parameters $s_{n}=1 /(n+2), \alpha_{n}=$ $1 /(n+2)^{2}$ for every $n \geq 0$. Fix $\mu=1, \lambda=1 / 2$, and $\gamma=1 / 10$. Take

$$
B=\left(\begin{array}{ccc}
1 & 0 & 1 \\
-1 & 1 & 0 \\
1 & 2 & -3
\end{array}\right), \quad b=\left(\begin{array}{c}
5 \\
-7 \\
-17
\end{array}\right)
$$

TABLE 1: Numerical results as regards Example 10.

\begin{tabular}{lcccc}
\hline$n$ & $x_{n}^{1}$ & $x_{n}^{2}$ & $x_{n}^{3}$ & $E_{n}$ \\
\hline 0 & 0.0000 & 1.0000 & 1.0000 & $6.63 E+00$ \\
50 & 1.8793 & -4.6691 & 3.1319 & $3.76 E-01$ \\
500 & 1.9875 & -4.9675 & 3.0125 & $3.70 E-02$ \\
2000 & 1.9969 & -4.9919 & 3.0031 & $9.21 E-03$ \\
\hline
\end{tabular}

The SFP can be formulated as the problem of finding a point $x^{*}$ with the property

$$
x \in C, \quad B x^{*} \in Q,
$$

where $C=H_{1}, Q=\{b\} \subset H_{2}$. That is, $x^{*}$ is the solution of system of linear equations $B x=b$, and

$$
x^{*}=\left(\begin{array}{c}
2 \\
-5 \\
3
\end{array}\right) \text {. }
$$

Then by Theorem 9 , the sequence $\left\{x_{n}\right\}$ is generated by

$$
\begin{aligned}
x_{n+1}= & \frac{1}{4(n+2)} x_{n} \\
& +\frac{n+1}{n+2}\left(x_{n}-\frac{1}{10} B^{*} B x_{n}+\frac{1}{10} B^{*} b-\frac{1}{10(n+2)^{2}} x_{n}\right) .
\end{aligned}
$$

As $n \rightarrow \infty$, we have $\left\{x_{n}\right\} \rightarrow x^{*}=(2,-5,3)^{T}$ (Table 1).

\section{Conflict of Interests}

The authors declare that there is no conflict of interests regarding the publication of this paper.

\section{Authors' Contribution}

All the authors read and approved the final paper.

\section{Acknowledgments}

The authors thank the referees for their helping comments, which notably improved the presentation of this paper. This work was supported by the Foundation of Tianjin Key Lab for Advanced Signal Processing and by the Fundamental Research Funds for the Central University (no. 3122014K012).

\section{References}

[1] H.-K. Xu, "Averaged mappings and the gradient-projection algorithm," Journal of Optimization Theory and Applications, vol. 150, no. 2, pp. 360-378, 2011.

[2] E.-S. Levitin and B.-T. Polyak, "Constrained minimization methods," Zhurnal Vychislitel'noi Matematiki i Matematicheskoi Fiziki, vol. 6, pp. 787-823, 1996.

[3] P. H. Calamai and J. J. Moré, "Projected gradient methods for linearly constrained problems," Mathematical Programming, vol. 39, no. 1, pp. 93-116, 1987. 
[4] B. T. Polyak, Introduction to Optimization, Optimization Software, New York, NY, USA, 1987.

[5] M. Su and H.-K. Xu, "Remarks on the gradient-projection algorithm," Journal of Nonlinear Analysis and Optimization, vol. 1, no. 1, pp. 35-43, 2010.

[6] A. Moudafi, "Viscosity approximation methods for fixed-points problems," Journal of Mathematical Analysis and Applications, vol. 241, no. 1, pp. 46-55, 2000.

[7] H.-K. Xu, "Viscosity approximation methods for nonexpansive mappings," Journal of Mathematical Analysis and Applications, vol. 298, no. 1, pp. 279-291, 2004.

[8] L.-C. Ceng, Q. H. Ansari, and J.-C. Yao, "Some iterative methods for finding fixed points and for solving constrained convex minimization problems," Nonlinear Analysis: Theory, Methods \& Applications, vol. 74, no. 16, pp. 5286-5302, 2011.

[9] G. Marino and H.-K. Xu, "Convergence of generalized proximal point algorithms," Communications on Pure and Applied Analysis, vol. 3, no. 4, pp. 791-808, 2004.

[10] G. Marino and H.-K. Xu, "A general iterative method for nonexpansive mappings in Hilbert spaces," Journal of Mathematical Analysis and Applications, vol. 318, no. 1, pp. 43-52, 2006.

[11] A. E. Al-Mazrooei, A. Latif, and J. C. Yao, "Solving generalized mixed equilibria, variational inequalities, and constrained convex minimization," Abstract and Applied Analysis, vol. 2014, Article ID 587865, 26 pages, 2014.

[12] Y. Censor and T. Elfving, "A multiprojection algorithm using Bregman projections in a product space," Numerical Algorithms, vol. 8, no. 2-4, pp. 221-239, 1994.

[13] C. Byrne, "A unified treatment of some iterative algorithms in signal processing and image reconstruction," Inverse Problems, vol. 20, no. 1, pp. 103-120, 2004.

[14] Y. Censor, T. Elfving, N. Kopf, and T. Bortfeld, "The multiplesets split feasibility problem and its applications for inverse problems," Inverse Problems, vol. 21, no. 6, pp. 2071-2084, 2005.

[15] Y. Censor, T. Bortfeld, B. Martin, and A. Trofimov, "A unified approach for inversion problems in intensity-modulated radiation therapy," Physics in Medicine and Biology, vol. 51, no. 10, pp. 2353-2365, 2006.

[16] H.-K. Xu, "A variable Krasnosel'skii-Mann algorithm and the multiple-set split feasibility problem," Inverse Problems, vol. 22, no. 6, pp. 2021-2034, 2006.

[17] H.-K. Xu, "Iterative methods for the split feasibility problem in infinite-dimensional Hilbert spaces," Inverse Problems, vol. 26, no. 10, Article ID 105018, 2010.

[18] M. Tian, "A general iterative algorithm for nonexpansive mappings in Hilbert spaces," Nonlinear Analysis: Theory, Methods \& Applications, vol. 73, no. 3, pp. 689-694, 2010.

[19] K. Goebel and W. A. Kirk, Topics in Metric Fixed Point Theory, Cambridge Studies in Advanced Mathematics, Cambridge University Press, 1990.

[20] K. Goebel and S. Reich, Uniform Convexity, Hyperbolic Geometry and Nonexpansive Mapping, vol. 83 of Monographs and Textbooks in Pure and Applied Mathematics, Marcel Dekker, New York, NY, USA, 1984.

[21] L.-C. Ceng, M.-M. Wong, A. Petruşel, and J.-C. Yao, "Relaxed implicit extragradient-like methods for finding minimumnorm solutions of the split feasibility problem," Fixed Point Theory, vol. 14, no. 2, pp. 327-344, 2013.
[22] L. C. Ceng, A. Petruşel, and J. C. Yao, "Relaxed extragradient methods with regularization for general system of variational inequalities with constraints of split feasibility and fixed point problems," Abstract and Applied Analysis, vol. 2013, Article ID 891232, 25 pages, 2013. 


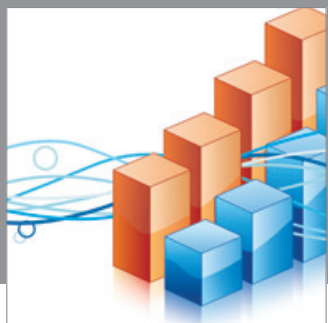

Advances in

Operations Research

mansans

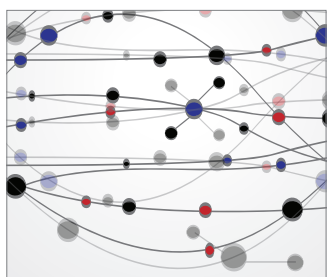

The Scientific World Journal
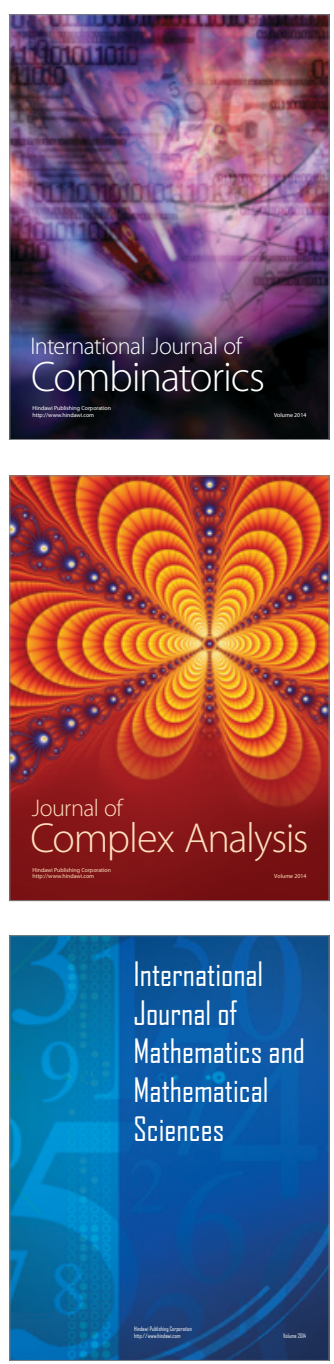
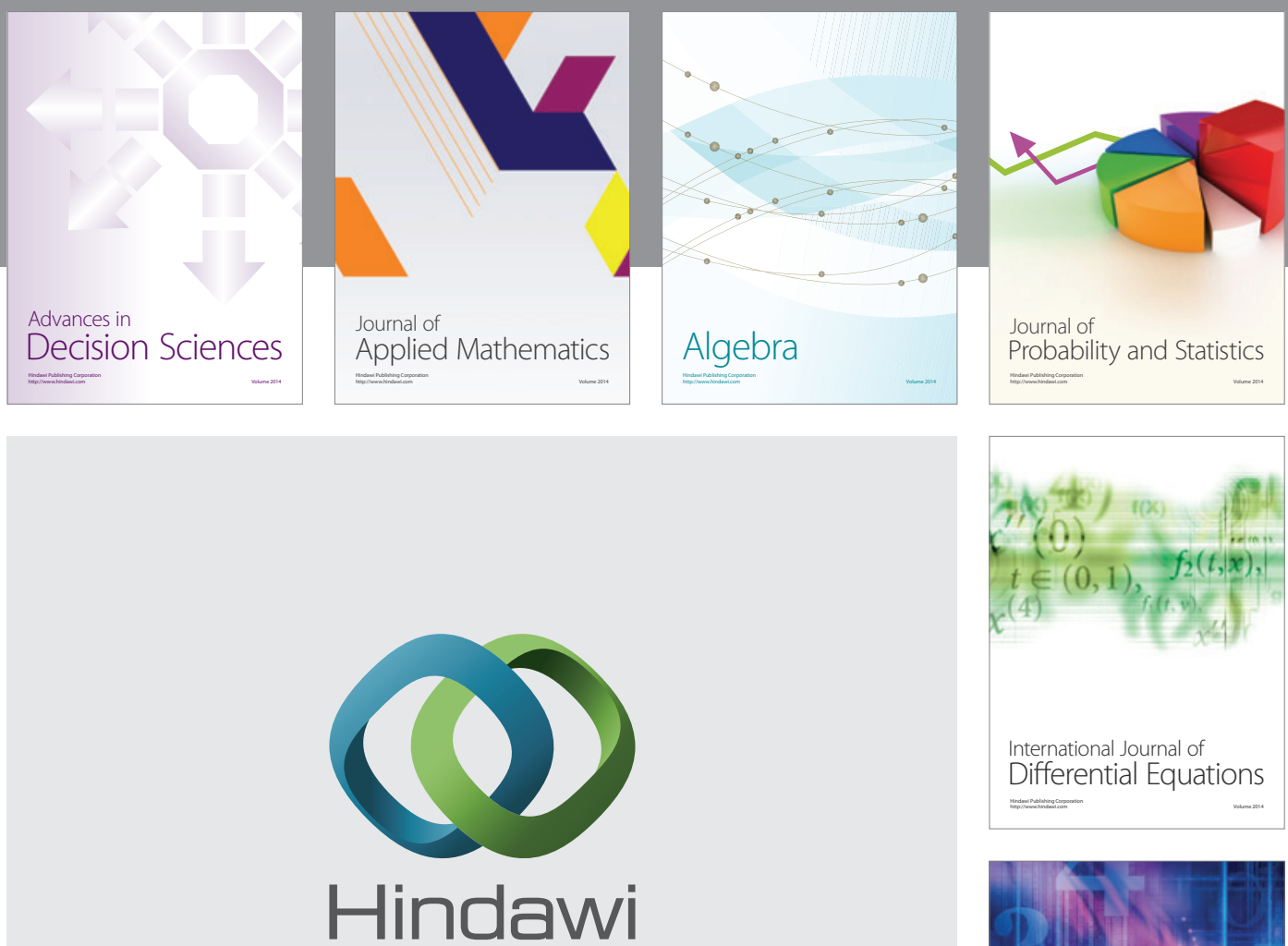

Submit your manuscripts at http://www.hindawi.com
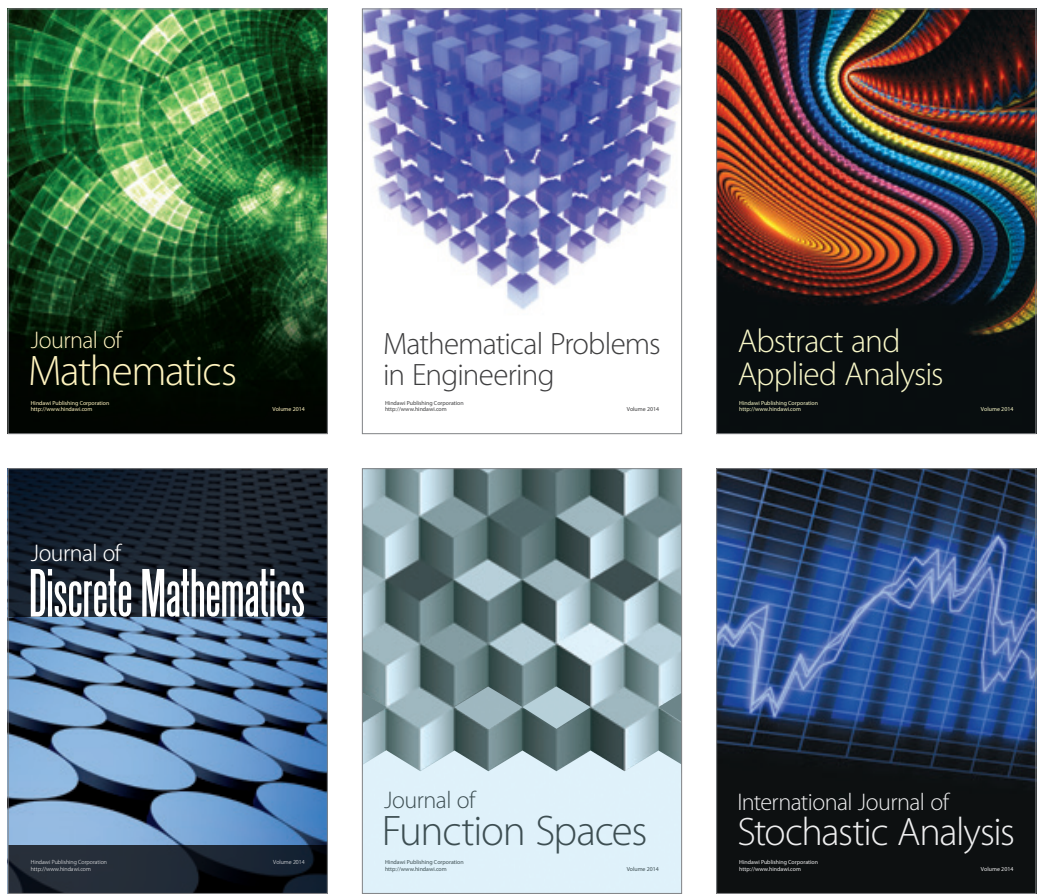

Journal of

Function Spaces

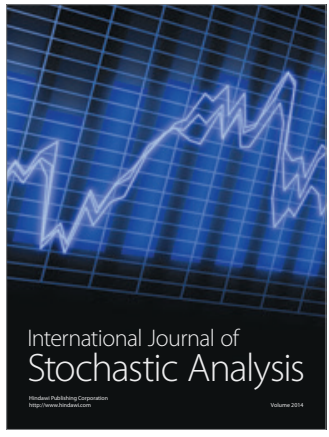

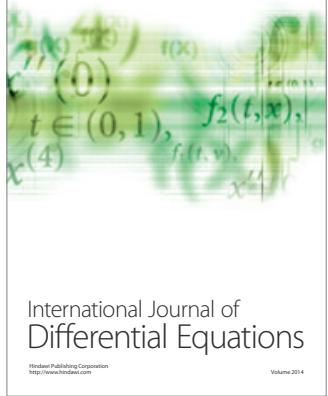
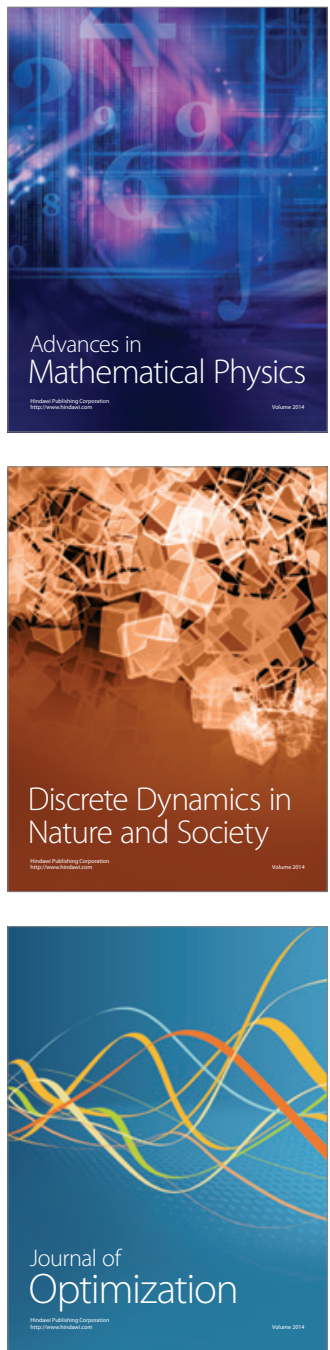